\title{
Malignant glomus tumor of the lung with multiorgan metastases: case report and literature review
}

This article was published in the following Dove Press journal:

OncoTargets and Therapy

28 July 2015

Number of times this article has been viewed

\author{
Liang-Liang Dong' \\ En-Guo Chen' \\ Irfan S Sheikh² \\ Zhi-Nong Jiang ${ }^{3}$ \\ Ai-Hua Huang ${ }^{3}$ \\ Ke-Jing Ying' \\ 'Department of Pulmonary Medicine, \\ ${ }^{2}$ Department of Internal Medicine, \\ Sir Run Run Shaw Hospital, \\ ${ }^{3}$ Department of Pathology, \\ Sir Run Run Shaw Hospital, College \\ of Medicine, Zhejiang University, \\ People's Republic of China
}

\begin{abstract}
Glomus tumor is an exceedingly rare neoplasm that is derived from cells of the neuromyoarterial glomus or glomus body. It rarely occurs in the visceral organs where glomus body may be sparse or even absent, such as the stomach, intestines, mediastinum, and respiratory tract. It is unusual for a glomus tumor to demonstrate atypical or malignant histopathological characteristics. It is also rare for such a tumor to express clinically aggressive behavior. However, when metastasis does occur,this disease is often fatal. We herein report an interesting case of a middle-age woman admitted due to progressive cough and hemoptysis. A polypoid mass was found to occlude the left lingular lobar bronchus. Final histopathologic examination showed the presence of malignant glomus tumor, confirmed by immunoreactivity for smooth muscle actin and vimentin. Two months later, the patient developed abdominal distension and gastrointestinal bleeding. Further evaluation lead to the discovery of widespread metastatic disease to the gastrointestinal tract, spleen, and the left adrenal gland. We further entail a review of the literature on the clinicopathologic features and diagnosis of this uncommon tumor.
\end{abstract}

Keywords: malignant glomus tumor, lung, metastasis, diagnosis, immunohistochemistry

\section{Introduction}

In general, glomus tumors are infrequent benign mesenchymal perivascular tumors,constituting approximately $1.6 \%$ of soft tissue tumors in one large study. ${ }^{1}$ The most common reported location is the distal extremities, particularly in the hand, wrist, foot, and the subungual region. Infrequently, it can also occur in internal organs such as the mediastinum, lung, trachea, and stomach. Primary pulmonary glomus tumors are extremely rare. Although predominantly benign, rarely glomus tumors may demonstrate aggressive and/or malignant clinical and histological features, such as nuclear atypia, infiltrative growth pattern, multicentricity. ${ }^{2}$ Till now, only 23 primary pulmonary glomus tumors have been reported, with seven cases displaying malignant characteristics. $^{3-8}$ Local invasiveness was observed in most of the reported cases with only one case exhibiting widespread metastasis. We herein report a case of a 60 -yearold female patient with a malignant glomus tumor of the lung with metastases to the gastrointestinal tract, spleen, and left adrenal gland. We also provide a review of the literature to raise awareness of this exceedingly rare tumor with an emphasis on describing the clinicopathological features with a detailed differential diagnoses.

\section{Case report}

A 60-year-old previously healthy female patient presented to our clinic complaining of recurrent cough and hemoptysis for 3 months. Physical examination did not reveal 
any significant findings. She was then followed with contrast computed tomography (CT), which revealed a $2.5 \times 1.5 \mathrm{~cm}$ broad-based enhancement at the lingual segment of the left upper border, near the hilum, with hilar lymphadenopathy (Figure 1A and B). Lung cancer was suspected as the most likely diagnosis.

Electronic bronchoscopy was then done revealing a white polypoid tumor, which occupied the left lingular bronchus without any signs of invasion or protrusion into the left main bronchus (Figure 2A). Biopsy of the tumor was taken and sent for pathology, which revealed inflammatory exudates and various degrees of necrosis. The patient once got a similar pathological report at another hospital where she had undergone bronchoscopic biopsy and lung puncture before arrival at our hospital. These inconclusive results were not enough to identify the final diagnosis. The patient was then discharged home but was lost to follow-up in 1 month.

Two months later, the patient presented again to our department with worsening of her symptoms. This time she reported a worsening of her cough and hemoptysis with a new onset of melena and abdominal distention. Physical examination revealed absence of breath sounds in the left lower lung field with consensual dullness to percussion and decreased vocal fremitus. Her abdominal and anal physical examinations were unremarkable. We performed another bronchoscopy, which showed the same white polypoid tumor with significant protrusion into the left main bronchus (Figure 2B). The tumor was then partly excised by high-frequency electric snare ligation under bronchoscopy and sent for pathological examination. An abdominal contrast CT was then performed, which demonstrated multiple mass-like lesions occupying the gastrointestinal cavity, spleen, and left adrenal gland (Figure 1C and D). The largest mass measured approximately $4.0 \times 3.0 \mathrm{~cm}$ in the left lower quadrant of the abdomen. The CT findings were consistent with metastatic disease, and the possibility of a malignant tumor was considered. An upper endoscopy was performed with ligation of mass lesions in the fundus, antrum, and duodenum (Figure 2C and D).

Pathology of the biopsy specimens revealed relatively uniform polygonal cells with oval to round nuclei, pale
A

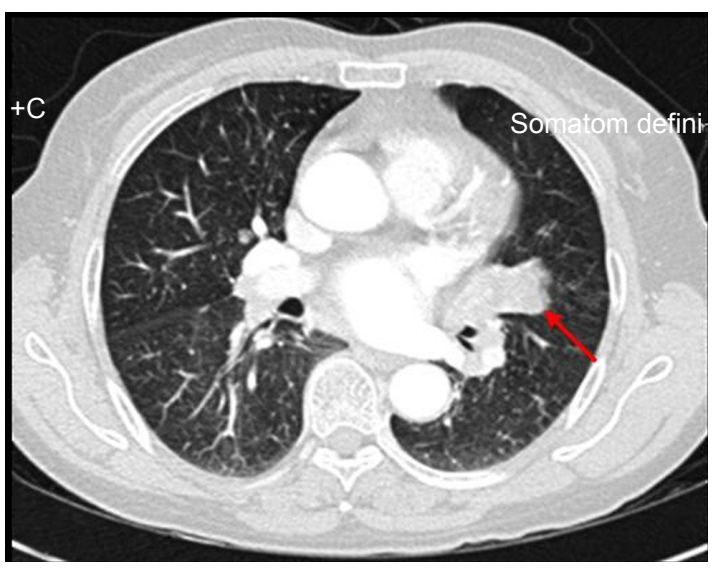

C

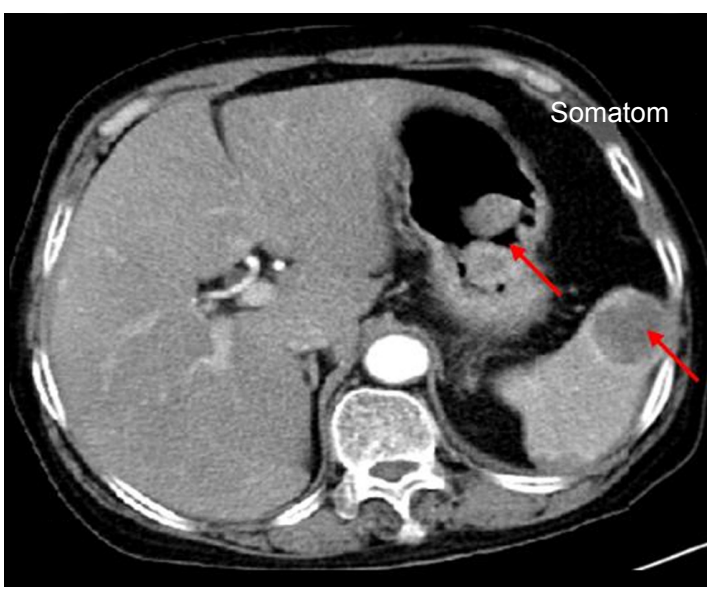

B

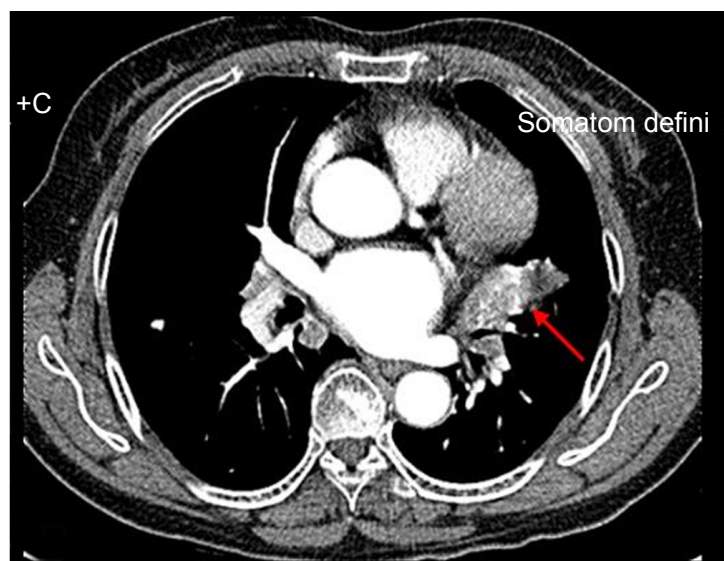

D

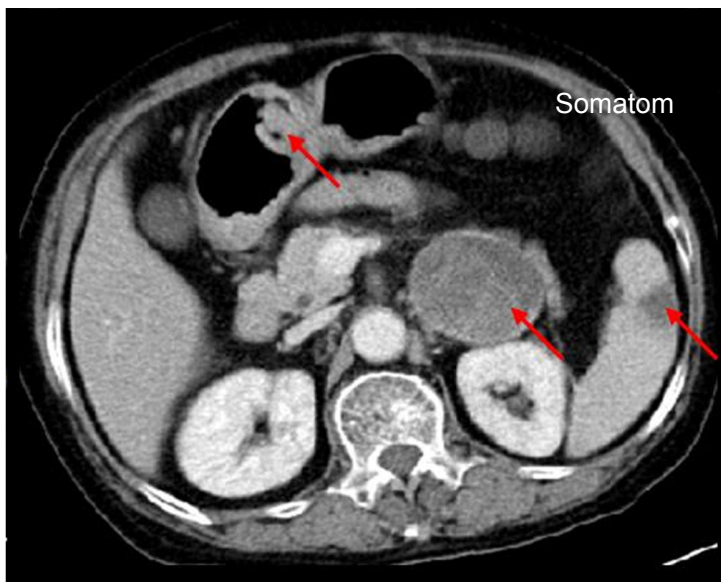

Figure I CT scan appearance.

Notes: (A and B) Chest computed tomography on hospital admission showed irregular mass in the lingual segment of upper lobe of left lung (arrows). (C and D) Abdomen CT scan demonstrated multiple masses occupying gastrointestinal cavity, spleen, and left adrenal gland (arrows).

Abbreviation: CT, computed tomography. 

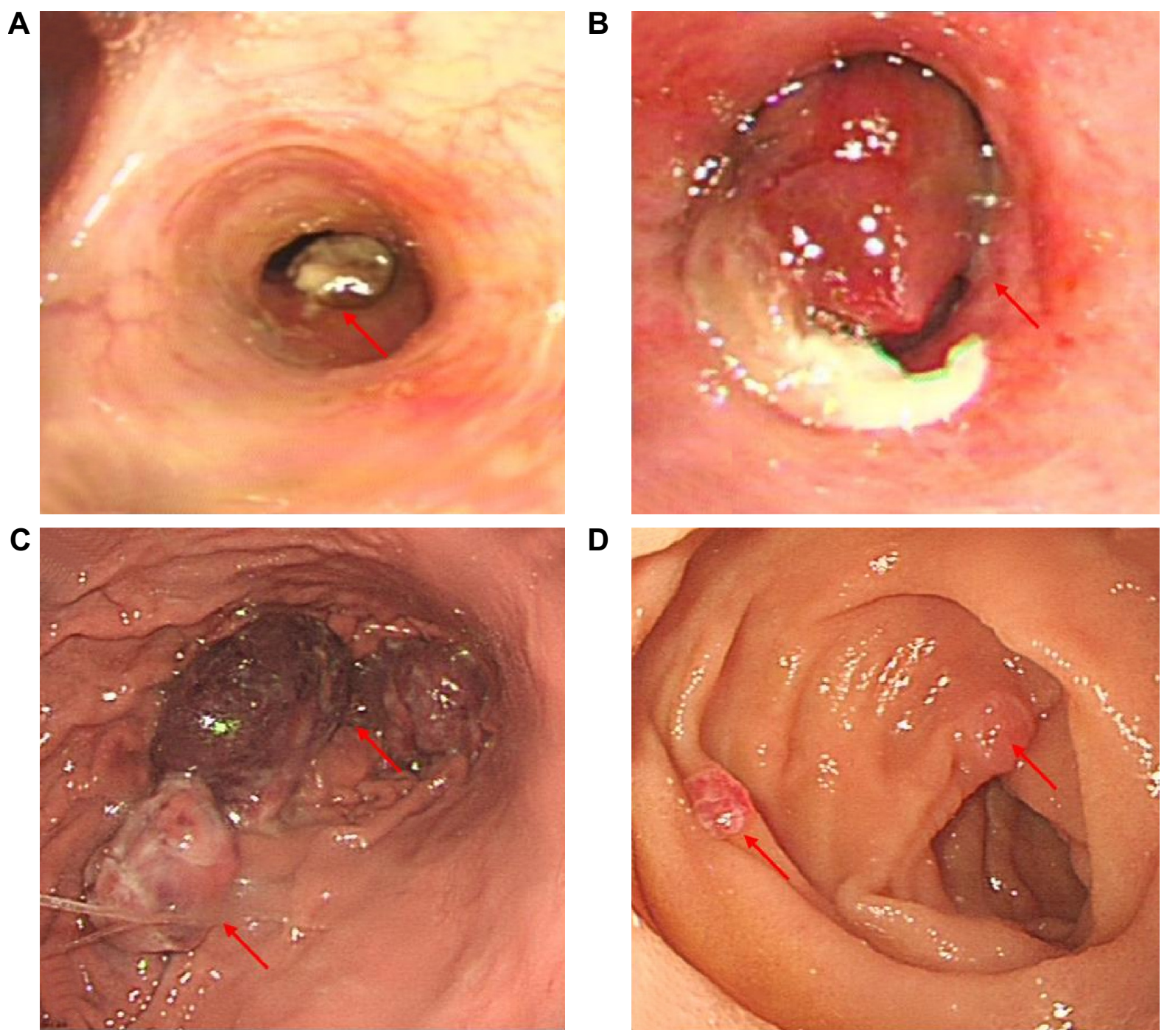

Figure 2 Findings from endoscopy.

Notes: (A) Bronchoscopy demonstrated a tumor mass in the left lingual bronchus (arrow). (B) The tumor projected into the left main bronchus on the second admission (arrow). (C) Gastroscopy demonstrated multiple submucosal masses in the fundus of the stomach (arrows). (D) Multiple lesions were also seen in the duodenum (arrows).

cytoplasm, and distinct cell borders that surrounded thin-walled blood vessels. Nuclear atypia, necrosis, and mitotic activity were also present. Staining for PAS was negative. Immunohistochemistry of the biopsy specimens obtained via bronchoscopy stained positive for smooth muscle actin and vimentin, and was partially positive for CD99 (Figure 3) and F8. Staining for cytokeratin, desmin, collagen IV, S100, CD117, and CD34 was negative. Immunohistochemistry of the specimens taken from the antrum and fundus stained positive for smooth muscle actin, CD31, and collagen IV, and was partially positive for $\mathrm{F} 8$ and $\mathrm{Ki}-67$. Staining for cytokeratin, desmin, S100, and CD117 was negative. The staining patterns observed confirmed the origin of the tumor to be that of smooth muscle. These findings supported the diagnosis of a malignant glomus tumor.
Due to the general poor condition of the patient, no chemotherapy was given. Within 1 month, the patient's symptoms worsened with massive hemoptysis. Bronchial arteriography revealed multiple areas of hemorrhage in the left lower lobes. Bronchial artery embolization was effectively performed; however, the gastrointestinal bleeding became intractable and nonresponsive to treatment. The patient died shortly of advanced multiorgan failure.

\section{Discussion}

Glomus tumors are relatively uncommon entities, with an estimated incidence of approximately $1.6 \%$ among soft tissue tumors. ${ }^{1}$ They usually occur in areas rich in glomus bodies such as the subungual regions of digits or the deep dermis of the palm, wrist, forearm, and foot. Infrequently, these entities 
A

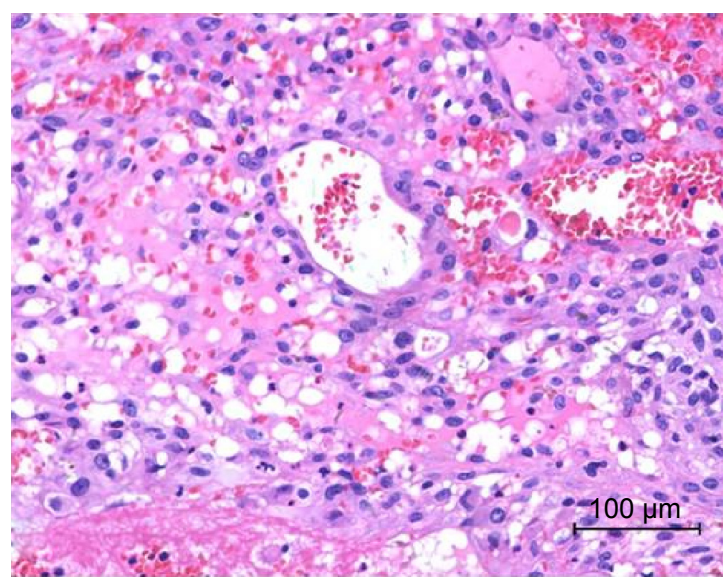

C

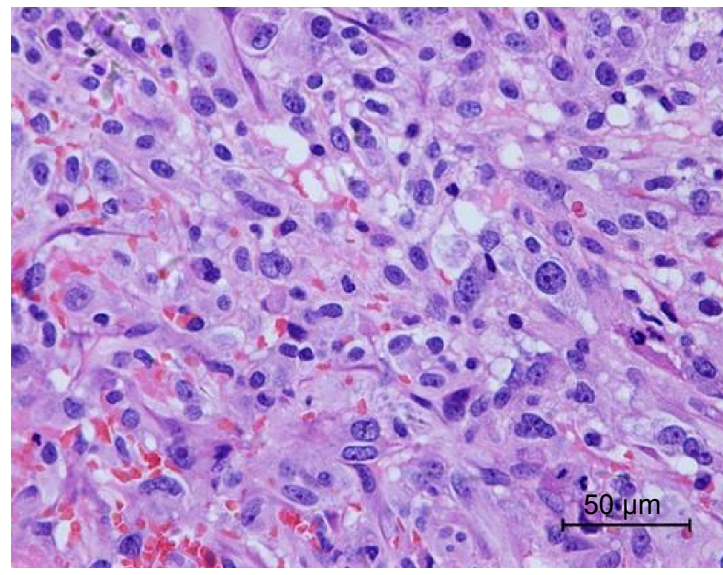

E

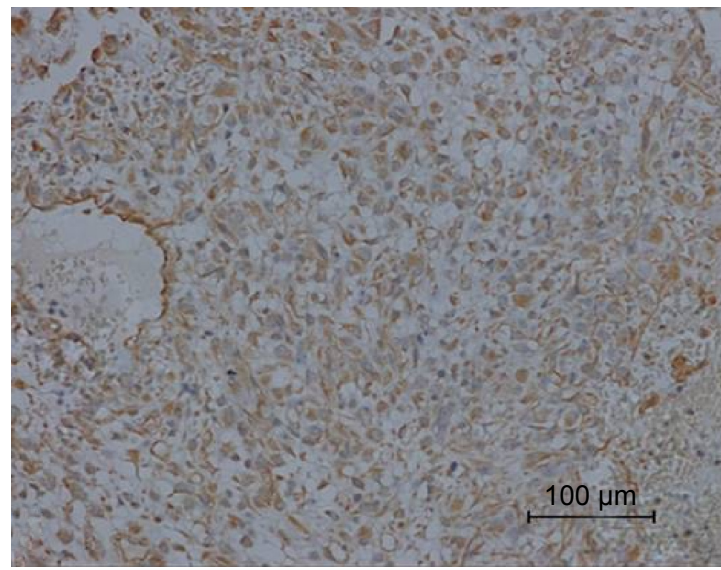

B

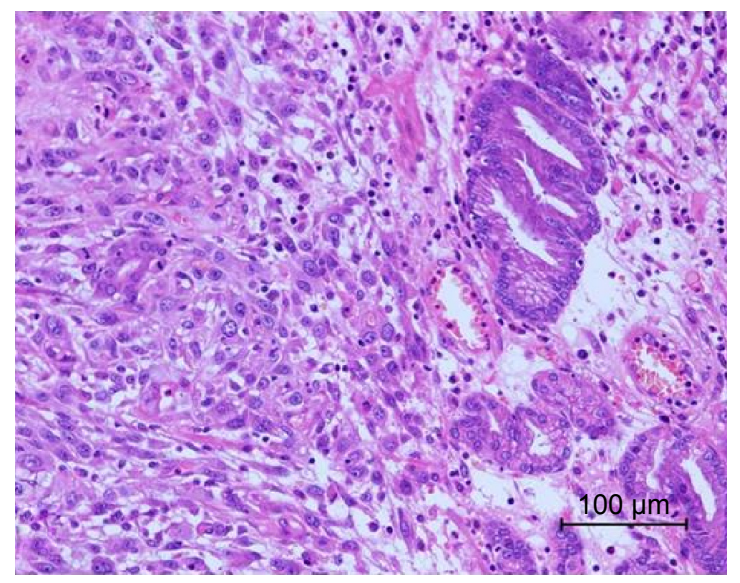

D

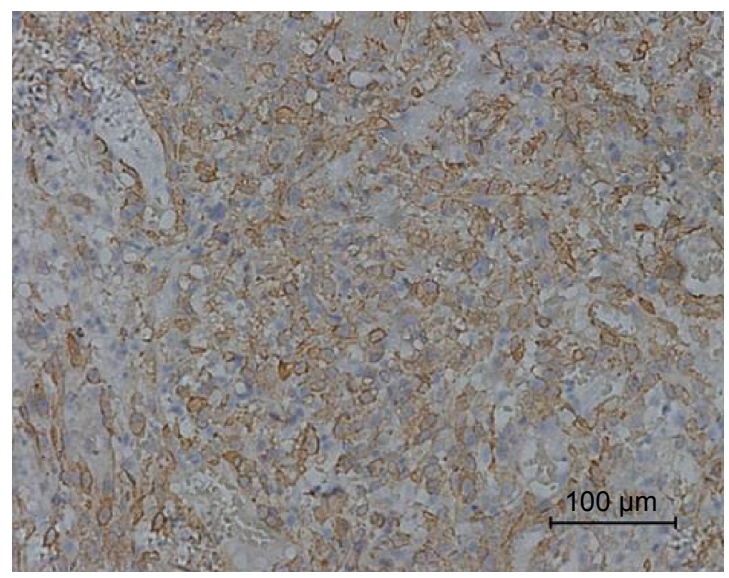

$\mathbf{F}$

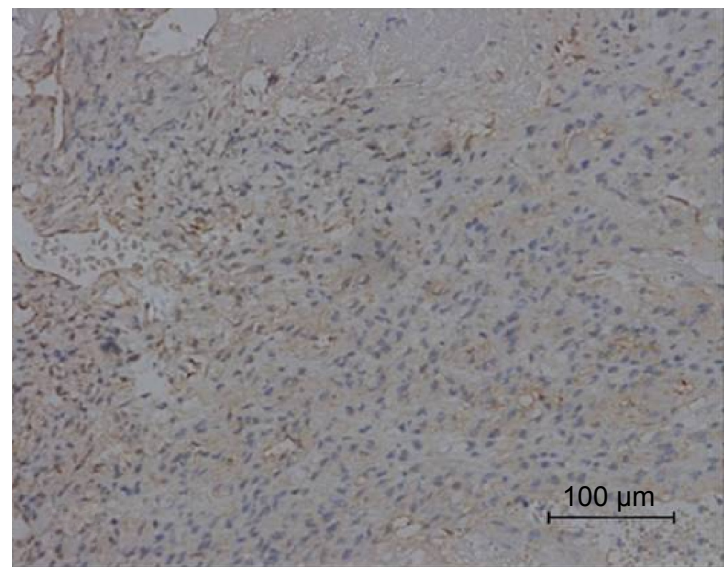

Figure 3 Microscopic appearances.

Notes: (A) Tumor cells of lung, adjacent to small vessels, were mostly oval to spindle with abundant pale cytoplasm and distinct cell borders and the tumor showed focal necrosis with inflammatory exudate (H\&E stain, 200X). (B) The metastatic tumor in gastric antrum reveals similar morphology (H\&E stain, 200X). (C) Marked nuclei and prominent nucleoli in present with mitotic activity (H\&E stain, 400x). (D-F) Immunohistochemistry: tumor cells of lung showed strong immunoreactivity for smooth muscle actin and vimentin, and it was partially positive for CD99 (200x).

Abbreviation: H\&E, hematoxylin and eosin.

have been observed in extracutaneous locations, where the normal glomus body may be sparse or even absent, such as the gastrointestinal, cardiovascular, respiratory tracts, and other visceral organs. The underlying mechanisms are equivocal. As such, diagnosis is often deferred or even missed. Most peripheral and visceral glomus tumors are histologically and clinically benign, but very rarely have they been considered to be malignant based on nuclear atypia, necrosis, elevated mitotic activity, and characteristically cystic degeneration. ${ }^{2}$

In 1972, the first report of a clinically atypical infiltrating glomus tumor was given by Lumley and Stansfeld. ${ }^{9}$ One year later, Anagnostou et al illustrated a case they claimed to be a pathologically malignant glomus tumor. ${ }^{10}$ Albeit 
the extremely low incidence, they suggested that glomus tumors might appear malignant based on certain pathological findings. In 1996, Brathwaite and Poppiti described a clinically malignant (ie, metastatic) glomus tumor in a patient with multiple glomus body hamartoma. ${ }^{11}$ Actually, the true malignant nature of glomus tumors has been a subject of emotive debate over many years. In 2001, Folpe et al examined 52 unusual glomus tumors previously diagnosed as "atypical" or "malignant" by virtue of nuclear atypia, infiltrative growth, or mitotic activity. They classified these lesions into four main types (malignant glomus tumor, symplastic glomus tumor, glomus tumor of uncertain malignant potential, and glomangiomatosis) and elaborated pathologic criteria for malignancy in these tumors. For malignant glomus tumor, it was defined as follows: tumors with a deep location and a size of more than $2 \mathrm{~cm}$, or atypical mitotic figures, or a combination of moderate to high nuclear grade and mitotic activity (five mitoses/50 HPF). There is a marked risk of metastasis and death from disease in patients whose tumor meets the above criteria. As for the present case, the lesion in the left lingular lobar bronchus was $2 \mathrm{~cm}$, and it was characterized by mitotic activity and distant metastasis. These findings appeared to be consistent with the diagnosis of malignant glomus tumor.

Malignant glomus tumors arising from the lung are extremely rare. To date, 23 primary pulmonary glomus tumors have been described in the literature, including only seven cases of malignant glomus tumor. Clinicopathologic features of reported cases, including our patient, are summarized in Table 1. The eight patients ranged from ages 9 years to 74 years with a median age of 50 years. It seems that malignant glomus tumors of lung had a male preponderance with a male to female ratio of $3: 1$. Two patients were incidentally detected during imaging for unrelated conditions and the remaining patients presented with nonspecific symptoms such as cough, hemoptysis, or chest pain. There are no reported cases in which the diagnosis was confirmed preoperatively. Folpe et al proposed that malignant glomus tumors are believed to be of low grade, locally aggressive sarcomas that rarely metastasize. Of the eight patients, six were positive for local invasion into adjacent lymph nodes and blood vessels. For malignant glomus tumors without widespread metastasis, the choice for surgical resection is potentially curative as seen in six cases. ${ }^{4}$ However, if there is widespread metastases, potential curative resection is unlikely, and the prognosis is dismal. The patient noted in one case report had received surgery and chemotherapy, but died 17 months later due to widespread metastases. Similar to the aforementioned case, our patient suffered from an outcome where there was widespread metastasis to the gastrointestinal tract, spleen, and adrenal glands. Our patient developed massive hemoptysis and gastrointestinal bleeding, which led to her death within a few months. For malignant glomus tumors, occurrence of distant metastasis may very readily result in fatality. Watanabe et al reported a case of malignant glomus tumors arising on the skin of the left hip that metastasized widely, leading to death. ${ }^{12}$ Folpe et al reported eight patients who developed metastatic disease and six were dead in $>3$ years. It was also stated that the time for the appearance of widespread metastasis is an average of 4 years postoperatively with a $29 \%$ mortality rate. ${ }^{3}$ From the literature review and as seen in our patient, glomus tumors have the potential to be metastatic and aggressive. Therefore, even after postresection of the primary tumor, close follow-up is strongly advocated due to the risk of metastasis.

The major differential diagnoses of malignant glomus tumors arising in the lung include carcinoid, haemangiopericytoma, smooth muscle neoplasms (especially epithelioid leiomyoma), paraganglioma, and primitive neuroectodermal

Table I Reported cases of primary malignant glomus tumors of lung

\begin{tabular}{|c|c|c|c|c|c|c|}
\hline References & Age (years), sex & Symptoms & Localization & Size $(\mathrm{cm})$ & Treatment & Follow-up \\
\hline Mackay et $\mathrm{al}^{7}$ & 19, Male & Incidential finding & Left lung & 2.5 & NA & NA \\
\hline Gaertner et $\mathrm{al}^{4}$ & 69 , Male & Hemoptysis & Right upper lobe & $9.5 \times 9 \times 5$ & Lobectomy; chemotherapy & DOD at 68 weeks \\
\hline Folpe et $\mathrm{al}^{3}$ & 38, Male & Incidential finding & Lung & 3.8 & NA & NA \\
\hline Folpe et $\mathrm{al}^{3}$ & 9, Female & Cough & Lung & 4.5 & NA & AWD at 60 months \\
\hline Hishida et $\mathrm{al}^{5}$ & 53 , Male & Cough & Right basal bronchus & $2.5 \times 2.0 \times 1.8$ & Right lower lobectomy & FOD at 23 months \\
\hline Kleontas et $\mathrm{al}^{6}$ & 74, Male & $\begin{array}{l}\text { Cough, dyspnea, } \\
\text { chest pain }\end{array}$ & Right upper lobe & $4.0 \times 2.6$ & Right upper lobectomy & FOD at 12 months \\
\hline Zhang et $\mathrm{al}^{8}$ & 48, Male & $\begin{array}{l}\text { Fever, cough, } \\
\text { hemoptysis }\end{array}$ & $\begin{array}{l}\text { Left upper lobe } \\
\text { and hilum }\end{array}$ & $3.5 \times 2.4 \times 2.0$ & Left upper lobectomy & DOOD at 4 days \\
\hline Current study & 60 , Female & $\begin{array}{l}\text { Cough, } \\
\text { hemoptysis }\end{array}$ & Left upper lobe & $2.5 \times 1.5$ & $\begin{array}{l}\text { Interventional } \\
\text { bronchoscopy }\end{array}$ & DOD at 6 months \\
\hline
\end{tabular}

Abbreviations: NA, not available; DOD, died of disease; FOD, free of disease; AWD, alive with disease; DOOD, died of other disease. 
tumors. Histological and immunohistochemical stainings can be effectively used to facilitate a definitive diagnosis of a given neoplastic lesion. Carcinoid tumors stain positively for keratin 18 , cytokeratin, chromogranin A, and synaptophysin. In contrast, glomus tumors are generally negative for the aforementioned markers. ${ }^{13}$ Hemangiopericytoma is another rare tumor that can present as an endobronchial mass and cause confusion with glomus tumor. Hemangiopericytomas stain negative for alpha-smooth muscle actin and are CD34 positive, which is a satisfactory evidence to rule out a diagnosis of pulmonary glomus tumor. ${ }^{14}$ Paragangliomas are typically composed of round epithelioid cells with small nuclei and stain substantially positive for neuroendocrine markers and S-100 protein. Other mimics of glomus tumor, such as smooth muscle tumors and liphomas, have exclusive immunohistochemical features and can be effortlessly identified. ${ }^{15}$

Our patient presented with pulmonary and stomach lesions that had similar cytological and immunohistochemical findings. Thus, the question arises as to the origin of the primary tumor. Although the pathologic characteristics of glomus tumor are occasionally described, there is little knowledge of specific immunohistochemical markers that are used to distinguish primary from secondary malignant glomus tumors. At first presentation, our patient complained of pulmonary symptoms without any abdominal disturbance. Bronchoscopy revealed a mass occupying the left lingular bronchus, which is not often seen in metastatic lung tumors. Also, the radiographic findings were not consistent with the diagnosis of metastatic lung cancer. All these aforementioned findings led to a final diagnosis of primary malignant glomus tumor of lung. Gastrointestinal metastasis of primary lung tumors is a highly uncommon event, and the mechanisms for this are unclear. ${ }^{16}$

In conclusion, we report a very unusual case of primary malignant tumor of the lung with aggressive course and extensive visceral involvement. Malignant glomus tumors of the lung are exceedingly rare, metastasis is even rarer. However, it should be considered as a differential when diagnosing other similar primary or metastatic lesions. Clinical examination, careful histological observation, and immunostaining for appropriate markers are essential in establishing

OncoTargets and Therapy

\section{Publish your work in this journal}

OncoTargets and Therapy is an international, peer-reviewed, open access journal focusing on the pathological basis of all cancers, potential targets for therapy and treatment protocols employed to improve the management of cancer patients. The journal also focuses on the impact of management programs and new therapeutic agents and protocols on the diagnosis of malignant glomus tumors. Currently, it is difficult for clinicians to accurately predict metastasis and prognosis of malignant glomus tumors of lung. Therefore, close follow-up of patients is imperative.

\section{Disclosure}

The authors report no conflicts of interest in this work.

\section{References}

1. Gombos Z, Zhang PJ. Glomus tumor. Arch Pathol Lab Med. 2008; 132(9):1448-1452.

2. Khoury T, Balos L, McGrath B, Wong MK, Cheney RT, Tan D. Malignant glomus tumor: a case report and review of literature, focusing on its clinicopathologic features and immunohistochemical profile. Am J Dermatopathol. 2005;27(5):428-431.

3. Folpe AL, Fanburg-Smith JC, Miettinen M, Weiss SW. Atypical and malignant glomus tumors: analysis of 52 cases, with a proposal for the reclassification of glomus tumors. Am J Surg Pathol. 2001;25(1):1-12.

4. Gaertner EM, Steinberg DM, Huber M, et al. Pulmonary and mediastinal glomus tumors - report of five cases including a pulmonary glomangiosarcoma: a clinicopathologic study with literature review. Am J Surg Pathol. 2000;24(8):1105-1114.

5. Hishida T, Hasegawa T, Asamura H, et al. Malignant glomus tumor of the lung. Pathol Int. 2003;53(9):632-636.

6. Kleontas A, Barbetakis N, Asteriou C, et al. Primary glomangiosarcoma of the lung: a case report. J Cardiothorac Surg. 2010;5:76.

7. Mackay B, Legha SS, Pickler GM. Coin lesion of the lung in a 19-yearold male. Ultrastruct Pathol. 1981;2(3):289-294.

8. Zhang Q, Wang S, Divakaran J, et al. Malignant glomus tumour of the lung. Pathology. 2010;42(6):594-596.

9. Lumley JS, Stansfeld AG. Infiltrating glomus tumour of lower limb. Br Med J. 1972;1(5798):484-485.

10. Anagnostou GD, Papademetriou DG, Toumazani MN. Subcutaneous glomus tumors. Surg Gynecol Obstet. 1973;136(6):945-950.

11. Brathwaite CD, Poppiti RJ Jr. Malignant glomus tumor. A case report of widespread metastases in a patient with multiple glomus body hamartomas. Am J Surg Pathol. 1996;20(2):233-238.

12. Watanabe K, Sugino T, Saito A, Kusakabe T, Suzuki T. Glomangiosarcoma of the hip: report of a highly aggressive tumour with widespread distant metastases. Br J Dermatol. 1998;139(6):1097-1101.

13. Yilmaz A, Bayramgurler B, Aksoy F, Tuncer LY, Selvi A, Uzman O. Pulmonary glomus tumour: a case initially diagnosed as carcinoid tumour. Respirology. 2002;7(4):369-371.

14. Park JH, Oh SH, Yang MH, Kim NI. Glomangiosarcoma of the hand: a case report and review of the literature. J Dermatol. 2003;30(11): 827-833.

15. NakajimaY,KoizumiK,HaraguchiS, etal.Locally infiltrative glomus tumor of the bronchus: a case report. Ann Thorac Cardiovasc Surg. 2010;16(2): 113-117.

16. Lin $\mathrm{HC}$, Yu CP, Lin HA, Lee HS. A case of lung cancer metastasized to the gastrointestinal anastomosis site where the primary gastric cancer was resected 17 years ago. Lung Cancer. 2011;72(2):255-257.

\section{Dovepress}

patient perspectives such as quality of life, adherence and satisfaction. The manuscript management system is completely online and includes a very quick and fair peer-review system, which is all easy to use. Visit http://www.dovepress.com/testimonials.php to read real quotes from published authors. 